\title{
Gaussian trajectory approach to dissipative phase transitions: The case of quadratically driven photonic lattices
}

\author{
Wouter Verstraelen $\odot,{ }^{1, *}$ Riccardo Rota $\odot,{ }^{2, \dagger}$ Vincenzo Savona ${ }^{\circ},{ }^{2}$ and Michiel Wouters ${ }^{1}$ \\ ${ }^{1}$ TQC, University of Antwerp, B-2610 Wilrijk, Belgium \\ ${ }^{2}$ Institute of Physics, Ecole Polytechnique Fédérale de Lausanne (EPFL), CH-1015 Lausanne, Switzerland
}

(Received 4 December 2019; accepted 29 April 2020; published 12 May 2020)

\begin{abstract}
We apply the Gaussian trajectories approach to the study of the critical behavior of two-dimensional dissipative arrays of nonlinear photonic cavities, in the presence of two-photon driving and in regimes of sizable loss rates. In spite of the highly mixed character of the density matrix of this system, the numerical approach is able to provide precise estimations of the steady-state expectation values, even for large lattices made of more than 100 sites. By performing a finite-size scaling of the relevant properties of the steady state, we extrapolate the behavior of the system in the thermodynamic limit and we show the emergence of a second-order dissipative phase transition, belonging to the universality class of thermal Ising model. This result indicates the occurrence of a crossover when the loss rate is increased from the weak-loss limit, in which the phase transition belongs to the universality class of the quantum Ising model.
\end{abstract}

DOI: 10.1103/PhysRevResearch.2.022037

Dissipative phase transitions are critical phenomena emerging in the nonequilibrium steady state of open quantum systems, due to the competition between their coherent Hamiltonian dynamics and the incoherent processes [1,2]. In recent years, the possibility of realizing strongly correlated states in photonic cavity arrays [3-5] has stimulated a deep investigation of these phenomena, which have been discussed theoretically in photonic systems [6-18], lossy polariton condensates [19-21], and spin models [22-30]. From the experimental point of view, remarkable results have been recently obtained with driven circuit quantum electrodynamics systems [31] and semiconductor microcavities [32,33], showing the possibility of observing dissipative phase transitions in real open quantum many-body systems.

The nontrivial interplay among the Hamiltonian evolution, driving, and dissipative processes in open quantum manybody systems has given rise to several fundamental questions about the respective roles of quantum and classical fluctuations across a dissipative phase transition. In this regard, the universality classes of these critical phenomena has been intensely debated [19,20,26,27,34-38]. In many cases, dissipative phase transitions emerge in regimes where the dissipation rates are comparable with the typical energy scale of the Hamiltonian and their universality classes belong to those of classical thermal phase transition $[27,36,39,40]$, such that

\footnotetext{
*Wouter.Verstraelen@uantwerpen.be

${ }^{\dagger}$ Riccardo.Rota@epfl.ch

Published by the American Physical Society under the terms of the Creative Commons Attribution 4.0 International license. Further distribution of this work must maintain attribution to the author(s) and the published article's title, journal citation, and DOI.
}

the critical behavior can be described in term of an effective temperature emerging as a results of dissipations.

In this debate, arrays of nonlinear photonic resonators in the presence of two-photon-i.e., quadratic in the fielddriving have recently attracted a certain attention, as these systems undergo a dissipative phase transition which belongs to a quantum universality class in a suitable regime of parameters [18]. Quadratically driven resonators have been realized experimentally with superconducting circuits [41,42] and, in recent years, several works have considered the possibility of exploiting the $\mathbb{Z}_{2}$ symmetry of this system in order to simulate the behavior of quantum spin systems, proposing also noiseresilient quantum codes based on this platform [43-47]. In the context of critical phenomena, the spontaneous breaking of the $\mathbb{Z}_{2}$ symmetry in a lattice of coupled quadratically driven resonators has been investigated with both mean-field [16] and many-body [18] approaches, showing the emergence of a phase transition in a regime where the loss rate is small compared to the Hamiltonian energy scales, with critical exponents equal to those of the quantum transverse Ising model.

In this Rapid Communication, we consider the behavior of a quadratically driven photonic lattice when the loss rate becomes comparable with the Hamiltonian parameters, showing that the universality class of the transition changes in this regime and one recovers the description in terms of a classical criticality. We perform this study using the Gaussian trajectories approach, a numerical technique introduced in the study of the single Kerr cavity with a one-photon pump [48]. This method turns out to be suitable to investigate dissipative phase transitions, as it allows us to estimate efficiently the dissipative dynamics of large two-dimensional lattices (of more than 100 sites) and, meanwhile, to include the relevant many-body correlations which arise in the vicinity of the critical point. Analyzing the finite-size scaling of the steady-state properties of the system, we estimate its behavior 
in the thermodynamic limit of infinitely large lattices and highlight the emergence of a second-order phase transition, with critical exponents equal to those of the classical Ising model.

A lattice of $N$ coupled photonic cavities can be described by a Bose-Hubbard model, whose Hamiltonian contains a local term $\hat{h}_{i}$ acting on the $i$ th cavity and a nonlocal term modeling the photon hopping between different cavities (we set $\hbar=1$ ):

$$
\hat{H}=\sum_{i=1}^{N} \hat{h}_{i}-\sum_{\langle i j\rangle} \frac{J}{z}\left(\hat{a}_{i}^{\dagger} \hat{a}_{j}+\hat{a}_{j}^{\dagger} \hat{a}_{i}\right) .
$$

Here, $\hat{a}_{i}\left(\hat{a}_{i}^{\dagger}\right)$ is the annihilation (creation) operator acting on the $i$ th site and the last sum runs over the nearest-neighbor pairs $\langle i j\rangle, J$ and $z$ being, respectively, the hopping strength and the coordination number. The local term can be written as

$$
\hat{h}_{i}=-\Delta \hat{a}_{i}^{\dagger} \hat{a}_{i}+\frac{U}{2} \hat{a}_{i}^{\dagger 2} \hat{a}_{i}^{2}+\frac{G}{2} \hat{a}_{i}^{\dagger 2}+\frac{G^{*}}{2} \hat{a}_{i}^{2},
$$

where $\Delta$ is the detuning between half of the two-photon driving field frequency and the resonant cavity frequency, $U$ is the photon-photon interaction energy associated to the Kerr nonlinearity, and $G$ is the two-photon driving field amplitude.

Assuming that the dissipative processes are Markovian, the dynamics of the open quantum system is recovered in terms of the density matrix $\hat{\rho}(t)$ which solves the Lindblad master equation:

$$
\frac{\partial \hat{\rho}}{\partial t}=\mathcal{L} \hat{\rho},=-i[\hat{H} \hat{\rho}]+\sum_{j, k} \hat{\Gamma}_{j, k} \hat{\rho} \hat{\Gamma}_{j, k}^{\dagger}-\frac{1}{2}\left\{\hat{\Gamma}_{j, k}^{\dagger} \hat{\Gamma}_{j, k} \hat{\rho}\right\} .
$$

Here, $\mathcal{L}$ is the Liouvillian superoperator and $\hat{\Gamma}_{j, k}$ are the jump operators which describe the coupling of the system with the environment: In general, the system exhibits local one- and two-photon losses, which are modelled respectively with the jump operators $\hat{\Gamma}_{1, j}=\sqrt{\gamma} \hat{a}_{j}$ and $\hat{\Gamma}_{2, j}=\sqrt{\eta} \hat{a}_{j}^{2}$. In particular, we will assume that the operators $\hat{\Gamma}_{1, j}$ ensure that the system relaxes toward a nonequilibrium steady state $\hat{\rho}_{S S}$, and the emergence of a persistent nonstationarity $[49,50]$ is avoided. Conditions for the existence of a unique steady state have been largely investigated in the literature [51,52].

Liouvillian $\mathcal{L}$ presents a $\mathbb{Z}_{2}$ symmetry coming from its invariance under a global change of sign of the annihilation operators, $\hat{a}_{i} \rightarrow-\hat{a}_{i} \forall i$. In the thermodynamic limit of an infinite lattice, the $\mathbb{Z}_{2}$ symmetry can be spontaneously broken for large values of $G / \gamma$ and $J / \gamma$, as indicated by a mean-field analysis [16]. This leads to the emergence of a transition between a phase with a $\mathbb{Z}_{2}$-symmetric steady state (i.e., $\operatorname{Tr}\left(\hat{\rho}_{S S} \sum_{i} \hat{a}_{i}\right)=0$ ) and a coherent phase with nonzero expectation value of the Bose field (i.e., $\operatorname{Tr}\left(\hat{\rho}_{S S} \sum_{i} \hat{a}_{i}\right) \neq 0$ ).

The nature of the dissipative phase transition can be investigated in terms of an approximate spin model, where two Schrödinger-cat states with opposite parity play the role of the two $s=1 / 2$ spin states with opposite magnetization [18]. When projecting the bosonic Hamiltonian in Eq. (1) onto the basis spanned by the Schrödinger-cat states, we recover the Hamiltonian of a quantum transverse $X Y$ model, where the photon-hopping term plays the role of an anisotropic spin coupling in the $x y$ plane (the coupling can be either ferromagnetic [18] or antiferromagnetic [53], according to the sign of $J$ ) and the detuning $\Delta$ plays the role of a transverse magnetic field in the $z$ direction. This model is known to present a phase transition belonging to the universality class of the quantum transverse Ising model [54].

From a computational perspective, the numerical solution of master equation (3) becomes intractable even for small lattices. The corner-space renormalization method [55] has been used to study quadratically driven-dissipative lattices with large nonlinearities and small loss rates, but it fails in the description of regimes characterized by a large photon occupancy per cavity and a highly mixed steady state, which are the regimes we consider here.

An alternative method which can overcome these difficulties is the Gaussian trajectories approach (GTA), which has been developed for the numerical simulation of polaritonic systems [48] and applied to study the temporal coherence of a dye-microcavity photon condensate [56]. As in an exact wave function Monte Carlo approach [57], the GTA recovers the density matrix $\hat{\rho}(t)$ of the open quantum system from the average of a set of $N_{T}$ pure states $\left|\psi_{n}(t)\right\rangle$ (usually called quantum trajectories) obtained independently by integrating a stochastic differential equation: $\hat{\rho}(t)=\frac{1}{N_{T}} \sum_{j=1}^{N_{T}}\left|\psi_{n}(t)\right\rangle\left\langle\psi_{n}(t)\right|$. The physical picture underlying this formalism is to consider the environment as a measurement apparatus continuously monitoring the open system, and hence the stochastic evolution of each quantum trajectory may be interpreted as the result of the different random outcomes of these measurements [58-61]. The peculiarity of the GTA approach is the Gaussian ansatz for the quantum trajectories, which makes the pure state $\left|\psi_{n}\right\rangle$ completely determined by its first and second central moments $[62,63]$. This assumption reduces notably the computational cost needed for the numerical integration of the stochastic differential equation, since the complexity of the problem within GTA scales quadratically with the number $N$ of lattice sites, rather than exponentially.

Contrary to the exact wave function Monte Carlo method, where there is no univocal choice of the stochastic differential equation for the trajectories $\left|\psi_{n}(t)\right\rangle$ in order to reproduce the same master equation for the density matrix $\hat{\rho}(t)$, the assumption of a Gaussian ansatz for $\left|\psi_{n}(t)\right\rangle$ makes the accuracy of the method dependent on the choice of the stochastic unravelings used to factorize the coupling of the quantum system with the external environment. For the single-site problem with quadratic driving and losses, it was shown in Ref. [64] that in the homodyne unraveling [65], exact trajectories remain nearly coherent states, a subclass of Gaussian states. Here, we will therefore use the heterodyne unraveling, a symmetrized version of homodyne detection. Heterodyne measurements measure the field quadratures with equal weights and are equivalent to two complementary homodyne detection schemes. It is a common procedure in quantum optics, where the photon losses are interfered with an out-of-resonance reference beam [65] and is mathematically closely connected to the stochastic collapse model known as quantum state diffusion (QSD) [66]. Interestingly, it has recently been suggested that such unraveling naturally captures macroscopic phenomena as phase transitions [67,68]. The derivation of the full stochastic differential equations for the dynamics of the quadratically driven-dissipative photonic 


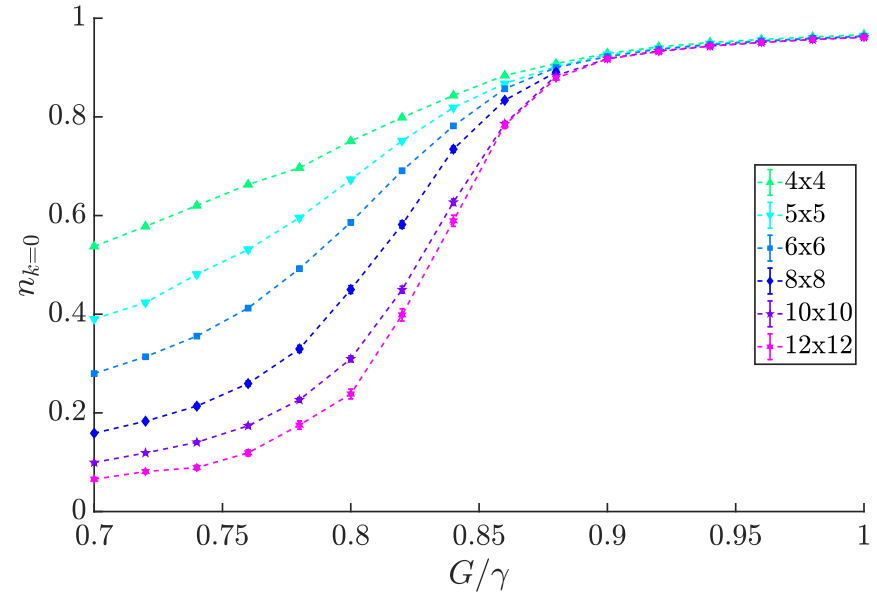

FIG. 1. The steady-state relative occupation of the $k=0$ mode increases as a function $G / \gamma$, as computed in lattices of different size. Results are obtained from 1000 trajectories (500 for the $12 \times 12$ lattice), each evolved over a total time $t=100 \gamma^{-1}$. For each quantum trajectory, the vacuum is chosen as initial state and the two-photon driving is switched on slowly to the desired value, in order to reduce the formation of defects which would appear from strong quenching [70]. The steady-state expectation values are computed averaging the results obtained in a time interval from a size-dependent initial time $t_{\text {relax }}$ to the final time $t_{\text {fin }}=100 \gamma^{-1}$.

lattice within the GTA can be found in the Supplemental Material [69].

Another aspect which makes the GTA suitable for the study of dissipative phase transitions is that the weak $\mathbb{Z}_{2}$ symmetry of the Liouvillian is not preserved in a single quantum trajectory, but it is restored only after averaging many trajectories. Therefore, it is possible to obtain nontrivial results in the calculation of a suitable order parameter along an individual trajectory at long times. The study of the distribution of the latter expectation values over the whole set of sampled trajectories is helpful to understand the emergence of collective phases in different regimes of the physical parameters, and hence provides an important insight into the critical behavior in the thermodynamic limit [29].

We apply the GTA formalism to the study of the quadratically driven dissipative Bose-Hubbard model in twodimensional (2D) square lattices of different size, with periodic boundary conditions. In the Supplemental Material [69], we show that the method is able to replicate the finding of quantum-critical behavior for parameters similar to those in Ref. [18], and furthermore a benchmark study that shows the accuracy of this method for a dimer. Since our main objective is to investigate the dissipative phase transition in regimes where the loss rates are comparable with the Hamiltonian parameters, we here set $U / \gamma=J / \gamma=1, \Delta=-J$. The last condition assures that the two-photon driving is resonant with the $k=0$ mode of the single-particle energy band of the closed system, and is useful to avoid any bistable behavior. Moreover, since two-photon losses are not expected to play an important role in the emergent criticality [18], we set $\eta=0$ : This choice allows for a relevant speed-up of the numerical calculations [69].
In Fig. 1, the steady-state expectation value for the relative occupation of the $k=0$ mode

$$
n_{k=0}=\frac{\sum_{j j^{\prime}} \operatorname{Tr}\left(\hat{\rho}_{S S} \hat{a}_{j}^{\dagger} \hat{a}_{j^{\prime}}\right)}{\left[\sum_{j} \operatorname{Tr}\left(\hat{\rho}_{S S} \hat{a}_{j}^{\dagger} \hat{a}_{j}\right)\right]^{2}}
$$

is plotted as a function of the driving amplitude $G$ for different lattice sizes. At large values of $G / \gamma$, we notice that $n_{k=0} \simeq 1$, independently of the size of the lattice. This result indicates that the steady state of the dissipative system in this limit is a coherent state characterized by long-range order, since the local Bose fields on each cavity assume the same value. This is in agreement with the picture of the spontaneous symmetry breaking obtained the mean-field calculation [16]. At smaller values of $G / \gamma, n_{k=0}$ decreases when the lattice size increases, suggesting that $n_{k=0} \rightarrow 0$ in the thermodynamic limit. This behavior supports the hypothesis of the presence of a disordered $\mathbb{Z}_{2}$-symmetric phase for small values of the driving amplitude.

Given this last result, it is convenient to describe the behavior of the system across the phase transition in terms of an order parameter, which is related to the average value of the Bose fields on the different cavities. We thus define $\bar{\alpha}=$ $\operatorname{Im}\left\langle\psi\left|\frac{1}{N} \sum_{i} \hat{a}_{i}\right| \psi\right\rangle$, i.e., the expectation value of the average Bose field on a single quantum trajectory (we consider its imaginary part in order to have a real-valued order parameter): This quantity has a clear physical meaning, as it corresponds to the outcome of a heterodyne unraveling in real experiments. In Figs. 2(a)-2(d), we plot the time evolution of $\bar{\alpha}$ on single trajectories in a $6 \times 6$ lattice, choosing the vacuum as the initial state at $t=0$, for different values of the driving amplitude. For $G=0.7 \gamma$ [Fig. 2(a)], we notice a behavior supporting the emergence of a disordered phase, as the order parameter exhibits Gaussian fluctuations around the value $\bar{\alpha}=0$. For $G=0.8 \gamma$ [Fig. 2(b)], the situation is similar, but we can see the presence of longer intervals of time where the value of $\bar{\alpha}$ stagnates around nonzero values, suggesting the appearance of a bimodal character in the steady-state distribution of the order parameter. At larger values of the driving amplitude $[G \geqslant 0.9 \gamma$, Figs. 2(c) and 2(d)], the behavior of $\bar{\alpha}(t)$ is completely different: After a first transient where the value of $\bar{\alpha}$ changes notably in time, at long times we see that the order parameter fluctuates around a nonzero value, which can be either negative [as in Fig. 2(c)] or positive [as in Fig. 2(d)], according to the particular realization of the noise in the stochastic differential equation. This behavior suggest the emergence of an ordered phase with broken symmetry in this regime of parameters. However, when considering an ensemble of many trajectories at given $G$, half of them will stabilize at a long time around a positive value for $\bar{\alpha}$ and the other half around the opposite value $-\bar{\alpha}$, retrieving thus a $\mathbb{Z}_{2}$-symmetric steady-state density matrix, as expected for a system of finite size. This behavior becomes clear from sampling $p(\bar{\alpha})$, the probability distribution of $\bar{\alpha}$, shown in Fig. 2(e). Although $p(\bar{\alpha})$ is always an even function, we notice that, when increasing $G$, the character of the distribution changes from monomodal to bimodal behavior.

A more quantitative analysis can be performed from the Binder cumulant, defined as $U_{L}=1-\mu_{4} /\left(3 \mu_{2}^{2}\right)$, where $\mu_{m}=\int d \bar{\alpha} \bar{\alpha}^{m} p(\bar{\alpha})$ denotes the $m$ th moment of the 

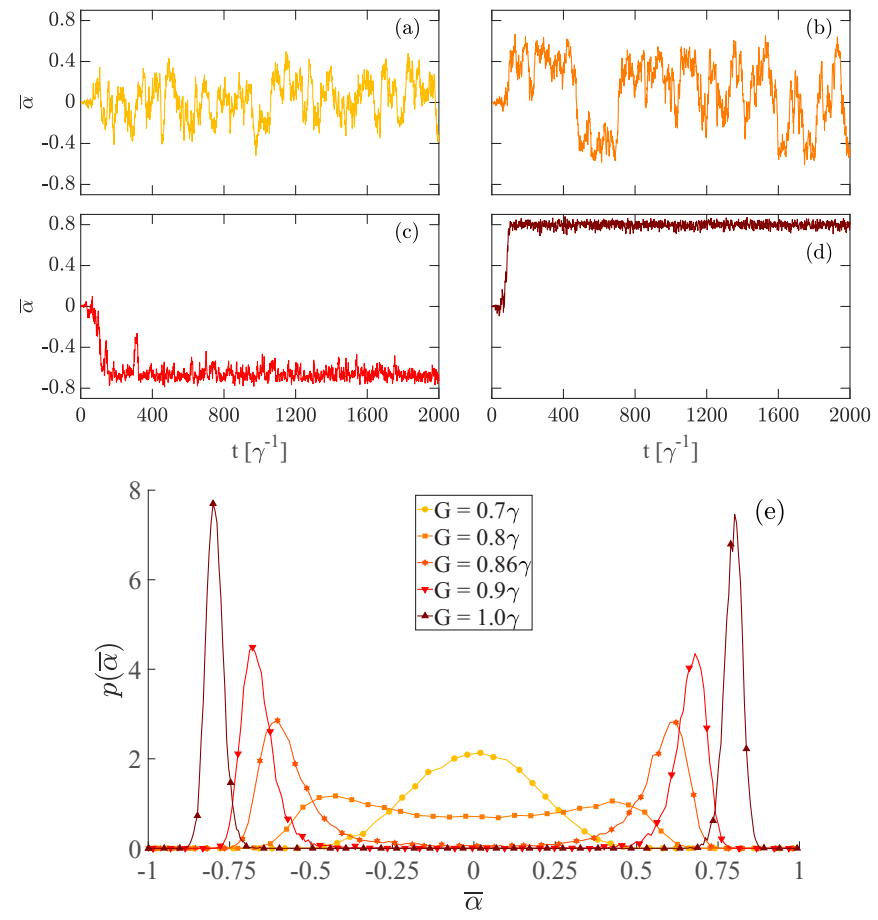

FIG. 2. [(a)-(d)] Time evolution of the order parameter $\bar{\alpha}$ along a single Gaussian trajectory in a $6 \times 6$ lattice, for different values of the driving amplitude: (a) $G / \gamma=0.7$, (b) $G / \gamma=0.8$, (c) $G / \gamma=0.9$, and (d) $G / \gamma=1.0$. (e) Probability distribution $p(\bar{\alpha})$ of the order parameter for different values of $G$. For each value of $G$, we consider 1000 trajectories each evolved over a time $t=400$ and we collect the data for $\bar{\alpha}(t)$ for $t \geqslant 20$, i.e., for long times in which the density matrix of the dissipative system has reached the steady state. The distribution $p(\bar{\alpha})$ is obtained as the histogram of the collected data. As $G$ increases, a qualitative transition from monomodal to bimodal distribution is evident.

probability distribution $p(\bar{\alpha})[71,72]$. This quantity has been deeply used in the finite-size scaling analysis of spin models in the presence of a paramagnetic-to-ferromagnetic phase transition, thanks to its peculiarity of being a universal function of the ratio $\xi / L$, with $\xi$ and $L$ being respectively the correlation length and the finite size of the lattice [71]. This means that at a critical point, where the correlation length diverges in the thermodynamic limit, the Binder cumulant becomes independent of the lattice size $L$ : Therefore, the finite-size scaling analysis of $U_{L}$ is a useful approach to track the emergence of the criticality in our system. In Fig. 3, we plot $U_{L}$ as a function of the driving amplitude $G$ for lattices of different sizes. We find that all the different curves cross at the common point $G_{c}=0.86 \gamma$, confirming the presence of a dissipative phase transition in our model. Some insight about the universality class of the phase transition can be obtained by plotting the same data for $U_{L}(\bar{\alpha})$ as a function of $\left(G-G_{c}\right) L^{1 / \nu}$, where $v$ is the critical exponent of the correlation length. From the results shown in Fig. 3 (inset), we notice that setting $v=1$ makes all the curves collapse, confirming the expected universal behavior of $U_{L}=f(\xi / L)$. The value $v=1$ corresponds to the critical exponent of the correlation length for a $2 \mathrm{D}$ classical Ising model. The

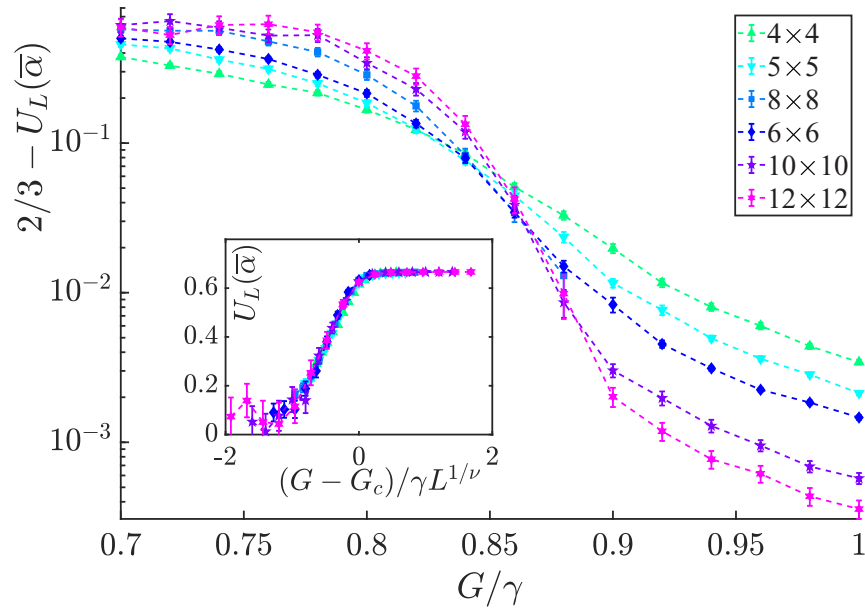

FIG. 3. Binder cumulant $U_{L}$ as a function of the two-photon driving amplitude $G$, for different lattice sizes $L$. A crossing of the curves for different lattice sizes can be seen, signaling the emergence of a critical point at $G=G_{c}$ (the difference $2 / 3-U_{L}$ is shown on a logarithmic scale). Inset: The curves for different sizes show an universal behavior when plotted as a function of $\left(G-G_{c}\right) L^{1 / \nu}$, with $v=1$ being the critical exponent for the correlation length in the classical 2D Ising model.

latter result indicates that the dissipative phase transition of a quadratically driven Bose-Hubbard model in the regime of large loss rates results from a classical criticality, contrary to what happens in regimes of small loss rate where the phase transition has a quantum nature [18].

The GTA method provides a simple picture of the crossover between quantum and classical critical behavior: Whereas quantum correlations between sites $n$ and $m$ are described by coefficients $u_{n m}$ and $v_{n m}$, classical correlations are given by the statistical correlator $\left\langle\alpha_{n} \alpha_{m}\right\rangle_{s}$, where $\langle\cdot\rangle_{s}$ denotes an average over trajectories. It is clear that strong dissipation has the same effect as strong measurements: It transfers quantum correlations to classical ones [66].

In this work, we have applied the Gaussian trajectory approach in the study of quadratically driven photonic lattices across a dissipative phase transition. This method turns out to be particularly suitable for theoretical analysis of critical phenomena in open quantum systems, as it provides accurate estimates of the physical observables even in regimes where the steady state is highly mixed.

In the case under consideration, we have been able to simulate lattices of up to 144 sites, which have allowed us to perform a precise finite-size scaling of the relevant properties of the system and to extract the critical exponent $v$ for the correlation length. The result $v=1$ is found, which differs from the value predicted by mean-field theory and corresponds to the classical Ising model, revealing the ability of the method to describe the many-body correlations arising among the photons. Furthermore, in the low-loss regime [69], we replicate the finding of the value $v=v_{q}$ of the quantum Ising model, which demonstrates that the method is able to capture the entanglement leading to relevant quantum correlations. This suggests a scenario in which, similar to the case at thermal equilibrium, a crossover between quantum 
and classical criticality in the nonequilibrium steady state occurs, depending on the scale of the loss rate relative to the Hamiltonian energy scale.

The possibility to use the Gaussian ansatz in the description of strongly correlated photons can open up several ways to apply our method in quantum many-body physics with light. An interesting perspective could be to use Gaussian trajectories to investigate the emergence of collective phenomena in photonic lattices in the presence of geometric frustration [53,73] or disorder [74]. Furthermore, the method is readily applicable to the study of the dynamics is these systems: Very recently, we have studied dynamical aspects of the same phase transition with the GTA method in Ref. [75].

Our case study to the quadratically driven Kerr lattice may also contribute to the general understanding of the crossover from quantum to classical critical behavior in dissipative phase transitions.

We acknowledge stimulating discussions with F. Minganti and D. Huybrechts. W.V. acknowledges financial support from the FWO Project No. 41190 and the FWO Travel Grant No. V413119N and the hospitality of LTPN.
[1] E. M. Kessler, G. Giedke, A. Imamoglu, S. F. Yelin, M. D. Lukin, and J. I. Cirac, Dissipative phase transition in a central spin system, Phys. Rev. A 86, 012116 (2012).

[2] F. Minganti, A. Biella, N. Bartolo, and C. Ciuti, Spectral theory of Liouvillians for dissipative phase transitions, Phys. Rev. A 98, 042118 (2018).

[3] I. Carusotto and C. Ciuti, Quantum fluids of light, Rev. Mod. Phys. 85, 299 (2013).

[4] M. J. Hartmann, Quantum simulation with interacting photons, J. Opt. 18, 104005 (2016).

[5] C. Noh and D. G. Angelakis, Quantum simulations and manybody physics with light, Rep. Prog. Phys. 80, 016401 (2016).

[6] H. J. Carmichael, Breakdown of Photon Blockade: A Dissipative Quantum Phase Transition in Zero Dimensions, Phys. Rev. X 5, 031028 (2015).

[7] J. J. Mendoza-Arenas, S. R. Clark, S. Felicetti, G. Romero, E. Solano, D. G. Angelakis, and D. Jaksch, Beyond mean-field bistability in driven-dissipative lattices: Bunching-antibunching transition and quantum simulation, Phys. Rev. A 93, 023821 (2016).

[8] W. Casteels, F. Storme, A. Le Boité, and C. Ciuti, Power laws in the dynamic hysteresis of quantum nonlinear photonic resonators, Phys. Rev. A 93, 033824 (2016).

[9] N. Bartolo, F. Minganti, W. Casteels, and C. Ciuti, Exact steady state of a Kerr resonator with one- and two-photon driving and dissipation: Controllable Wigner-function multimodality and dissipative phase transitions, Phys. Rev. A 94, 033841 (2016).

[10] M. Benito, C. Sánchez Muñoz, and C. Navarrete-Benlloch, Degenerate parametric oscillation in quantum membrane optomechanics, Phys. Rev. A 93, 023846 (2016).

[11] W. Casteels and C. Ciuti, Quantum entanglement in the spatialsymmetry-breaking phase transition of a driven-dissipative Bose-Hubbard dimer, Phys. Rev. A 95, 013812 (2017).

[12] W. Casteels, R. Fazio, and C. Ciuti, Critical dynamical properties of a first-order dissipative phase transition, Phys. Rev. A 95, 012128 (2017).

[13] M. Foss-Feig, P. Niroula, J. T. Young, M. Hafezi, A. V. Gorshkov, R. M. Wilson, and M. F. Maghrebi, Emergent equilibrium in many-body optical bistability, Phys. Rev. A 95, 043826 (2017).

[14] M. Biondi, G. Blatter, H. E. Türeci, and S. Schmidt, Nonequilibrium gas-liquid transition in the driven-dissipative photonic lattice, Phys. Rev. A 96, 043809 (2017).

[15] A. Biella, F. Storme, J. Lebreuilly, D. Rossini, R. Fazio, I. Carusotto, and C. Ciuti, Phase diagram of incoherently driven strongly correlated photonic lattices, Phys. Rev. A 96, 023839 (2017).

[16] V. Savona, Spontaneous symmetry breaking in a quadratically driven nonlinear photonic lattice, Phys. Rev. A 96, 033826 (2017).

[17] F. Vicentini, F. Minganti, R. Rota, G. Orso, and C. Ciuti, Critical slowing down in driven-dissipative Bose-Hubbard lattices, Phys. Rev. A 97, 013853 (2018).

[18] R. Rota, F. Minganti, C. Ciuti, and V. Savona, Quantum Critical Regime in a Quadratically Driven Nonlinear Photonic Lattice, Phys. Rev. Lett. 122, 110405 (2019).

[19] L. M. Sieberer, S. D. Huber, E. Altman, and S. Diehl, Dynamical Critical Phenomena in Driven-Dissipative Systems, Phys. Rev. Lett. 110, 195301 (2013).

[20] L. M. Sieberer, S. D. Huber, E. Altman, and S. Diehl, Nonequilibrium functional renormalization for driven-dissipative BoseEinstein condensation, Phys. Rev. B 89, 134310 (2014).

[21] E. Altman, L. M. Sieberer, L. Chen, S. Diehl, and J. Toner, Two-Dimensional Superfluidity of Exciton Polaritons Requires Strong Anisotropy, Phys. Rev. X 5, 011017 (2015).

[22] T. E. Lee, H. Häffner, and M. C. Cross, Antiferromagnetic phase transition in a nonequilibrium lattice of Rydberg atoms, Phys. Rev. A 84, 031402(R) (2011).

[23] T. E. Lee, S. Gopalakrishnan, and M. D. Lukin, Unconventional Magnetism Via Optical Pumping of Interacting Spin Systems, Phys. Rev. Lett. 110, 257204 (2013).

[24] C.-K. Chan, T. E. Lee, and S. Gopalakrishnan, Limit-cycle phase in driven-dissipative spin systems, Phys. Rev. A 91, 051601(R) (2015).

[25] J. Jin, A. Biella, O. Viyuela, L. Mazza, J. Keeling, R. Fazio, and D. Rossini, Cluster Mean-Field Approach to the Steady-State Phase Diagram of Dissipative Spin Systems, Phys. Rev. X 6, 031011 (2016).

[26] M. F. Maghrebi and A. V. Gorshkov, Nonequilibrium manybody steady states via Keldysh formalism, Phys. Rev. B 93, 014307 (2016).

[27] R. Rota, F. Storme, N. Bartolo, R. Fazio, and C. Ciuti, Critical behavior of dissipative two-dimensional spin lattices, Phys. Rev. B 95, 134431 (2017).

[28] V. R. Overbeck, M. F. Maghrebi, A. V. Gorshkov, and H. Weimer, Multicritical behavior in dissipative Ising models, Phys. Rev. A 95, 042133 (2017).

[29] R. Rota, F. Minganti, A. Biella, and C. Ciuti, Dynamical properties of dissipative $X Y Z$ Heisenberg lattices, New J. Phys. 20, 045003 (2018). 
[30] D. Roscher, S. Diehl, and M. Buchhold, Phenomenology of first-order dark-state phase transitions, Phys. Rev. A 98, 062117 (2018).

[31] J. M. Fink, A. Dombi, A. Vukics, A. Wallraff, and P. Domokos, Observation of the Photon-Blockade Breakdown Phase Transition, Phys. Rev. X 7, 011012 (2017).

[32] S. R. K. Rodriguez, W. Casteels, F. Storme, N. Carlon Zambon, I. Sagnes, L. Le Gratiet, E. Galopin, A. Lemaître, A. Amo, C. Ciuti, and J. Bloch, Probing a Dissipative Phase Transition Via Dynamical Optical Hysteresis, Phys. Rev. Lett. 118, 247402 (2017).

[33] T. Fink, A. Schade, S. Höfling, C. Schneider, and A. Imamoglu, Signatures of a dissipative phase transition in photon correlation measurements, Nat. Phys. 14, 365 (2018).

[34] S. Diehl, A. Tomadin, A. Micheli, R. Fazio, and P. Zoller, Dynamical Phase Transitions and Instabilities in Open Atomic Many-Body Systems, Phys. Rev. Lett. 105, 015702 (2010).

[35] E. G. Dalla Torre, E. Demler, T. Giamarchi, and E. Altman, Quantum critical states and phase transitions in the presence of non-equilibrium noise, Nat. Phys. 6, 806 (2010).

[36] E. G. Dalla Torre, E. Demler, T. Giamarchi, and E. Altman, Dynamics and universality in noise-driven dissipative systems, Phys. Rev. B 85, 184302 (2012).

[37] U. C. Täuber and S. Diehl, Perturbative Field-Theoretical Renormalization Group Approach to Driven-Dissipative BoseEinstein Criticality, Phys. Rev. X 4, 021010 (2014).

[38] J. Marino and S. Diehl, Driven Markovian Quantum Criticality, Phys. Rev. Lett. 116, 070407 (2016).

[39] A. Mitra, S. Takei, Y. B. Kim, and A. J. Millis, Nonequilibrium Quantum Criticality in Open Electronic Systems, Phys. Rev. Lett. 97, 236808 (2006).

[40] E. G. Dalla Torre, S. Diehl, M. D. Lukin, S. Sachdev, and P. Strack, Keldysh approach for nonequilibrium phase transitions in quantum optics: Beyond the Dicke model in optical cavities, Phys. Rev. A 87, 023831 (2013).

[41] Z. Leghtas, S. Touzard, I. M. Pop, A. Kou, B. Vlastakis, A. Petrenko, K. M. Sliwa, A. Narla, S. Shankar, M. J. Hatridge, M. Reagor, L. Frunzio, R. J. Schoelkopf, M. Mirrahimi, and M. H. Devoret, Confining the state of light to a quantum manifold by engineered two-photon loss, Science 347, 853 (2015).

[42] C. Wang, Y. Y. Gao, P. Reinhold, R. W. Heeres, N. Ofek, K. Chou, C. Axline, M. Reagor, J. Blumoff, K. M. Sliwa, L. Frunzio, S. M. Girvin, L. Jiang, M. Mirrahimi, M. H. Devoret, and R. J. Schoelkopf, A Schrödinger cat living in two boxes, Science 352, 1087 (2016).

[43] F. Minganti, N. Bartolo, J. Lolli, W. Casteels, and C. Ciuti, Exact results for Schrödinger cats in driven-dissipative systems and their feedback control, Sci. Rep. 6, 26987 (2016), article.

[44] H. Goto, Universal quantum computation with a nonlinear oscillator network, Phys. Rev. A 93, 050301(R) (2016).

[45] H. Goto, Bifurcation-based adiabatic quantum computation with a nonlinear oscillator network, Sci. Rep. 6, 21686 (2016).

[46] S. E. Nigg, N. Lörch, and R. P. Tiwari, Robust quantum optimizer with full connectivity, Sci. Adv. 3, e1602273 (2017).

[47] S. Puri, C. K. Andersen, A. L. Grimsmo, and A. Blais, Quantum annealing with all-to-all connected nonlinear oscillators, Nat. Commun. 8, 15785 (2017).
[48] W. Verstraelen and M. Wouters, Gaussian quantum trajectories for the variational simulation of open quantum-optical systems, Appl. Sci. 8, 1427 (2018).

[49] B. Buca, J. Tindall, and D. Jaksch, Non-stationary coherent quantum many-body dynamics through dissipation, Nat. Commun. 10, 1730 (2019).

[50] V. V. Albert, B. Bradlyn, M. Fraas, and L. Jiang, Geometry and Response of Lindbladians, Phys. Rev. X 6, 041031 (2016).

[51] H. Spohn, Approach to equilibrium for completely positive dynamical semigroups of $n$-level systems, Rep. Math. Phys. 10, 189 (1976); An algebraic condition for the approach to equilibrium of an open $n$-level system, Lett. Math. Phys. 2, 33 (1977).

[52] D. Nigro, On the uniqueness of the steady-state solution of the Lindblad-Gorini-Kossakowski-Sudarshan equation, J. Stat. Mech. (2019) 043202.

[53] R. Rota and V. Savona, Simulating frustrated antiferromagnets with quadratically driven QED cavities, Phys. Rev. A 100 , 013838 (2019).

[54] A. Dutta, G. Aeppli, B. K. Chakrabarti, U. Divakaran, T. F. Rosenbaum, and D. Sen, Quantum Phase Transitions in Transverse Field Spin Models: From Statistical Physics to Quantum Information (Cambridge University Press, Cambridge, UK, 2015).

[55] S. Finazzi, A. Le Boité, F. Storme, A. Baksic, and C. Ciuti, Corner-Space Renormalization Method for Driven-Dissipative Two-Dimensional Correlated Systems, Phys. Rev. Lett. 115, 080604 (2015).

[56] W. Verstraelen and M. Wouters, Temporal coherence of a photon condensate: A quantum trajectory description, Phys. Rev. A 100, 013804 (2019).

[57] K. Mølmer, Y. Castin, and J. Dalibard, Monte Carlo wavefunction method in quantum optics, J. Opt. Soc. Am. B 10, 524 (1993).

[58] S. Haroche and J.-M. Raimond, Exploring the Quantum: Atoms, Cavities, and Photons (Oxford University Press, Oxford, UK, 2013).

[59] H. Carmichael, Statistical Methods in Quantum Optics 2. Non-classical Fields, Theoretical and Mathematical Physics (Springer, Berlin, 2007)

[60] H. Wiseman and G. Milburn, Quantum Measurement and Control (Cambridge University Press, Cambridge, UK, 2010).

[61] A. J. Daley, Quantum trajectories and open many-body quantum systems, Adv. Phys. 63, 77 (2014).

[62] C. Weedbrook, S. Pirandola, R. García-Patrón, N. J. Cerf, T. C. Ralph, J. H. Shapiro, and S. Lloyd, Gaussian quantum information, Rev. Mod. Phys. 84, 621 (2012).

[63] A. Ferraro, S. Olivares, and M. Paris, Gaussian States in Quantum Information, Napoli Series on Physics and Astrophysics (Bibliopolis, Napoli, 2005).

[64] N. Bartolo, F. Minganti, J. Lolli, and C. Ciuti, Homodyne versus photon-counting quantum trajectories for dissipative Kerr resonators with two-photon driving, Eur. Phys. J.: Spec. Top. 226, 2705 (2017).

[65] C. Gardiner and P. Zoller, Quantum Noise: A Handbook of Markovian and Non-Markovian Quantum Stochastic Methods with Applications to Quantum Optics, Springer Series in Synergetics (Springer, Berlin, 2004).

[66] H.-P. Breuer and F. Petruccioni, The Theory of Open Quantum Systems (Oxford University Press, Oxford, UK, 2002). 
[67] W. T. Strunz and I. C. Percival, Classical mechanics from quantum state diffusion: A phase-space approach, J. Phys. A: Math. Gen. 31, 1801 (1998).

[68] V. Link, K. Luoma, and W. T. Strunz, Revealing the nature of nonequilibrium phase transitions with quantum trajectories, Phys. Rev. A 99, 062120 (2019).

[69] See Supplemental Material at http://link.aps.org/supplemental/ 10.1103/PhysRevResearch.2.022037 for the full GTA equations, a benchmark of the numerical performance, a reproduction of the phase transition in the quantum regime, and benchmark studies on a dimer.

[70] A. del Campo and W. H. Zurek, Universality of phase transition dynamics: Topological defects from symmetry breaking, Int. J. Mod. Phys. A 29, 1430018 (2014).
[71] K. Binder, Finite size scaling analysis of Ising model block distribution functions, Z. Phys. B 43, 119 (1981).

[72] K. Binder, Critical Properties from Monte Carlo Coarse Graining and Renormalization, Phys. Rev. Lett. 47, 693 (1981).

[73] M. Biondi, G. Blatter, and S. Schmidt, Emergent light crystal from frustration and pump engineering, Phys. Rev. B 98, 104204 (2018).

[74] F. Vicentini, F. Minganti, A. Biella, G. Orso, and C. Ciuti, Optimal stochastic unraveling of disordered open quantum systems: Application to driven-dissipative photonic lattices, Phys. Rev. A 99, 032115 (2019).

[75] W. Verstraelen and M. Wouters, Classical critical dynamics in quadratically driven Kerr resonators, Phys. Rev. A 101, 043826 (2020). 